\title{
Zur Lokomotionsaktivität des Amphipoden Orchestia platensis im Freiland und im Laboratorium
}

\author{
GEORG RüPPELL \\ Zoologisches Institut der Universität Kiel, Lebrstubl für Ökologie, \\ Kiel
}

\begin{abstract}
On the locomotion activity of the amphipod Orchestia platensis in the field and in the laboratory. The daily locomotory activity of Orchestia platensis (KRöYER) was recorded in situ and under constant conditions in the laboratory. The equipment for automatical recording is described. The species is very abundant along the coast of the Baltic Sea (non-tidal conditions). It only propagates for about half a year (April-October, Bock 1967). During this time the adults have a nocturnal activity; thus the time of ecological activity is very limited. Not every night suits Orchestia: severe darkness, rain, low temperature and wind, depress their activity. As a result, only about $10 \%$ of the total life-span of Orchestia can be utilized for feeding, propagation and other ecologically important functions. Further difficulties arise from catastrophies in the biotope of Orchestia: inundation or desiccation cause mass emigrations even during the day, when predators are active. Therefore losses are very high. Orchestia adapts to these conditions by at once utilizing favourable conditions. An endogenous component of their activity can be easily revealed by studies under constant conditions but is practically of no importance in the natural biotope. An endogenous pattern of activity, as in mammals or birds, is therefore not discernible.
\end{abstract}

\section{EINLEITUNG}

Die Frage nach der Aktivitätszeit eines Tieres ist von großer ökologischer Bedeutung. Nur ein aktives Tier wirkt im Haushalt seines Lebensraumes. Vielfach bietet ein Milieu für nur kurze Zeit im Tagesrhythmus Bedingungen, die einer Art aktives Leben gestatten. Damit stellt sich die Frage: Wie kurz darf diese Zeitspanne sein, um für die Erhaltung der Art zu genügen? Oft bieten die im Tagesgang wechselnden Faktoren verschiedenen Arten in zeitlicher Trennung günstige Lebensmöglichkeiten. Daher ist es erforderlich, beschriebene Nahrungskreisläufe anhand der Aktivitätszeiten zu überprüfen (REMMERT 1965). Verschiedene Individuen einer Art haben meist gleiche Aktivitätszeiten, wodurch ein Zusammentreffen der Individuen zur Fortpflanzung gewährleistet ist.

$\mathrm{Da} ß$ die Aktivitätszeit eines Tieres nicht nur durch die wechselnden Außenfaktoren, sondern gleichzeitig durch die innere Uhr gesteuert wird, ist in neuerer Zeit immer wieder hervorgehoben worden. Aufgabe der vorliegenden Studie ist es, bei dem Amphipoden Orchestia platensis (KRÖYER) den oben dargestellten Fragen nachzugehen und 
das Verhältnis zwischen exogenen und endogenen Faktoren zu studieren. Zugleich sollte die alte These geprüft werden, nach der phylogenetisch junge Landtiere nachtaktiv sind.

\section{MATERIAL UND METHODEN}

Für eine automatische Registrierung wurde eine Bodenfalle konstruiert, bei der 24 formolgefüllte Fächer einmal pro Tag unter einer Trichteröffnung vorbeigedreht werden. Ahnliche Fallen wurden auch von Tongiorgr (1963) und Williams (1958) entwickelt. Vielfach blieben die Fallen mehrere Tage hintereinander am gleichen Ort. Nur für die direkten Vergleiche aufeinanderfolgender Tage wurden sie täglich geleert. Bei dicht besiedelten Stränden spielte das Wegfangen der Tiere im Verlauf einiger Tage keine Rolle. Die Untersuchungen wurden an der Ostsee (Schleimünde), also in einem Gebiet ohne Gezeiten durchgeführt. Für die Auswertung wurden nur adulte Tiere verwendet, da sich junge anders verhalten.

Die Angaben aus den einzelnen Fangzeiten (April bis Oktober, einige Winterwerte) sind nur qualitativ miteinander vergleichbar, da die Beschaffenheit des Lebensraumes (Sandstrand mit Anwurf) dauernden Schwankungen unterworfen ist und damit auch die Besiedlungsdichte. Die so erhaltenen Freilanddaten wurden mit den gleichzeitig registrierten meteorologischen Daten verglichen. So konnte der Einfluß exogener Faktoren studiert werden. Für die Bedeutung endogener Faktoren wurde im Labor bei konstanter Temperatur ein künstliches Substrat aufgebaut und darin eine Population von Orchestia platensis gehalten. Ihre Lokomotionsaktivität wurde mit Hilfe einer federleichten Wippe gemessen, deren Ausschläge auf einer rotierenden Trommel aufgetragen wurden (Abb. 1). Ober einen Vergleich zwischen dieser Populationsaktivität und der von Einzeltieren wird an anderer Stelle berichtet werden.

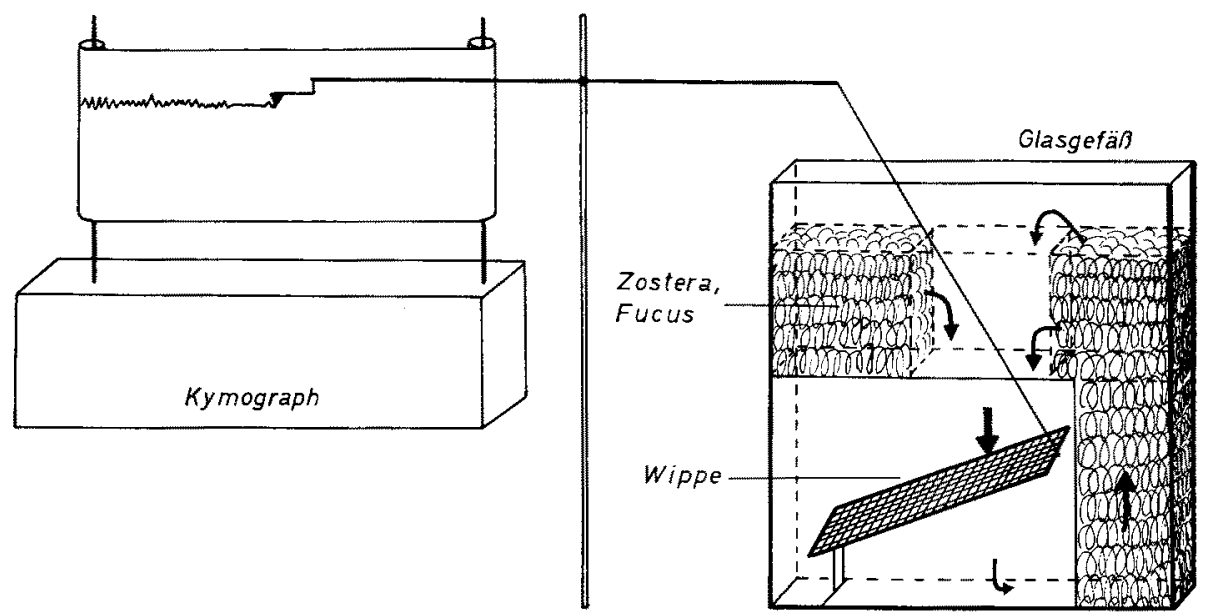

Abb. 1: Aktograph für automatische Registrierung der lokomotorischen Aktivität von Populationen des Amphipoden Orchestia platensis. In einer Klimakammer wird ein natürliches Substrat in der Weise aufgebaut, daß die in dem Substrat kletternden Tiere immer wieder auf die Wippe gelangen, deren Bewegungen von einem Kymographen aufgezeichnet werden. 


\section{ERGEBNISSE}

\section{Untersuchungen im Freiland}

In der Regel sind alle Talitriden nachtaktiv. Vergleichsmessungen an Talorchestia deshayesii im gleichen Gebiet, an Orchestia gammarellus auf Helgoland und an Talitrus saltator in List auf Sylt zeigten das gleiche Ergebnis. Geringe nächtliche Beleuchtungsstärken (Mondschein) hatten erheblich fördernden Einfluß auf die Gesamtaktivität von

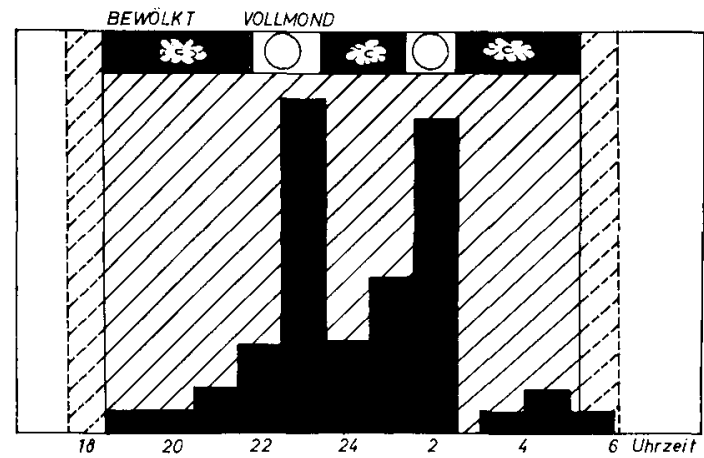

Abb. 2: Aktivitätsanstieg bei Orchestia platensis während der Dunkelperiode (schraffiert) durch Mondschein. Absinken der Aktivität, wenn eine geschlossene Wolkendecke den Mond verdeckt

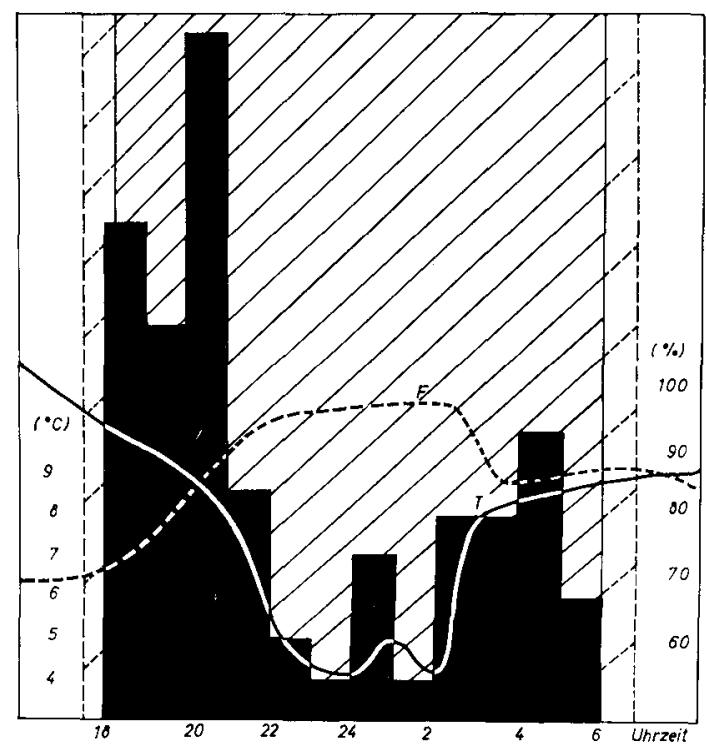

Abb. 3: Absinken der lokomotorischen Aktivität von Orchestia bei Temperaturen unter $5^{0} \mathrm{C}$; die Aktivitätskurve folgt im Grenzbereich sehr genau der Temperaturkurve 
Orchestia platensis (Abb. 2). Eine bedeutende Rolle spielte die Feuchtigkeit, wie bei Orchestia als thalassogenem Immigranten nicht anders zu erwarten ist. Die Luftfeuchtigkeit ist nachts in der Regel hoch genug (70\% relative Feuchtigkeit) und stellt damit keinen begrenzenden Faktor dar. Größeren Schwankungen unterliegt dagegen die Feuchtigkeit des Substrates, die infolge hoher Temperaturen stark absinken kann. Das führt zu wesentlich geringer Bewegungsaktivität der Tiere auf der Sandoberfläche. Ebenso wird die Aktivität durch niedrige Temperaturen (Abb. 3), durch hohe Windgeschwindigkeiten und durch heftigen Niederschlag herabgesetzt.

Nicht in jeder Nacht werden die Lebensansprüche von Orchestia voll erfüllt; viele Nächte bieten für diese Art ungünstige Bedingungen. Damit stehen Orchestia weniger als 10 bis $20 \%$ der gesamten Zeit zur Aktivität zur Verfügung (Dunkelheit zur Fortpflanzungsperiode von Orchestia = etwa $30 \%$ der Gesamtzeit). Trotz dieser relativ geringen Zeitspanne vermag Orchestia platensis sehr hohe Populationsdichten zu entwickeln.

Eine ungünstige Wirkung haben im Lebensraum dieser Art gelegentliche Bedingungen, die auch während der täglichen Ruhezeit Bewegung auslösen. Hier sind vor

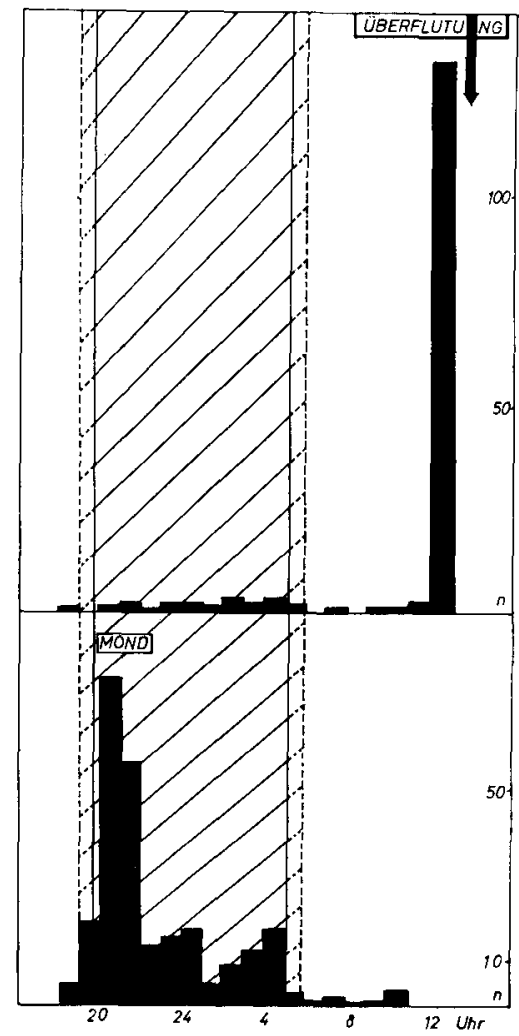

Abb. 4: Normale nächtliche Aktivität von Orchestia (unten), wobei mehrstündiger Mondschein (20-22 Uhr) aktivitätsfördernd wirkt. Am vorhergehenden Tag (oben) Katastrophenaktivität infolge kurzzeitiger Überflutung des Lebensraumes vom Meer her gegen $13 \mathrm{Uhr}$ 


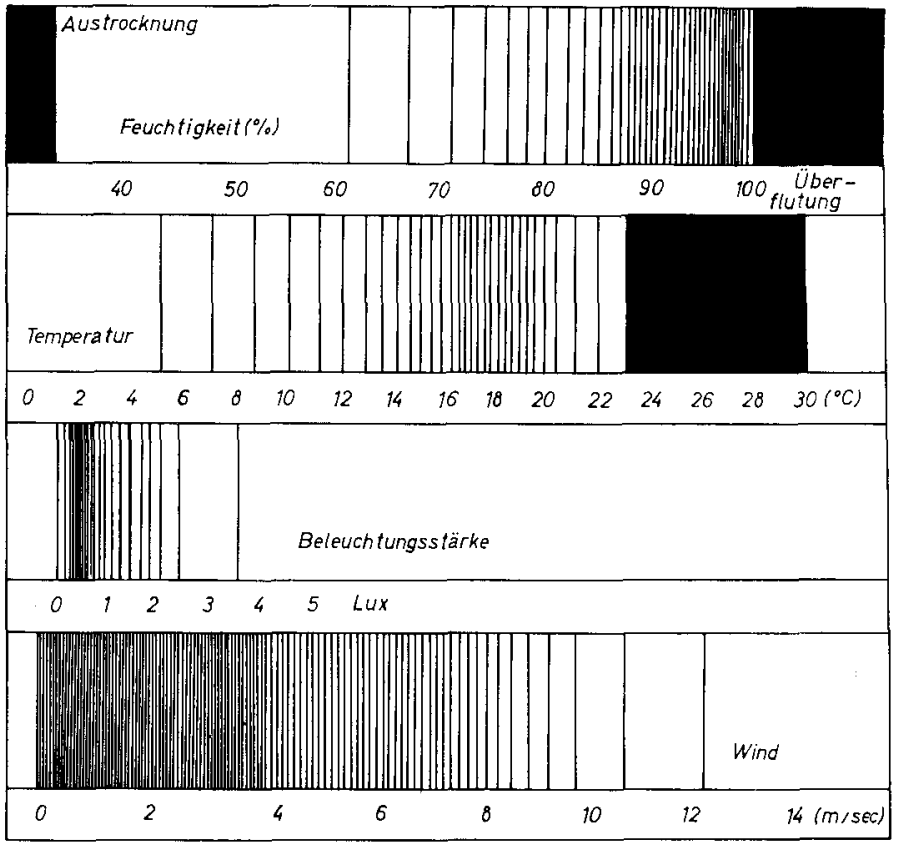

Abb. 5: Abhängigkeit der lokomotorischen Aktivität und ihrer Stärke bei Orchestia platensis von verschiedenen Faktoren (Feuchtigkeit, Temperatur, Beleuchtungsstärke, Windstärke). WeiBer Bereich: keine Aktivität möglich; eng schraffiert: Optimalbereich; schwarz: Katastrophenaktivität

allem extreme Austrocknungen und Überflutungen des Wohnsubstrates zu nennen. Dann können die Tiere in großer Zahl ihr Wohnsubstrat verlassen und neue, günstige Lebensräume suchen. Diese „Katastrophenaktivität“ kann zu sehr hohen Verlusten führen (Abb. 4); denn der Strand ist ein beliebtes Nahrungsrevier vieler Vögel.

Für die Aktivität von Orchestia platensis am Sandstrand ist also das Zusammentreffen einer Reihe von Faktoren notwendig. Der am weitesten vom Optimum entfernte Faktor bestimmt dabei die Höhe und das Auftreten von Aktivität überhaupt (Abb. 5). Insofern ist die Aktivität von Orchestia direkt von der Umwelt abhängig; durchgehende Aktivitätsmuster, wie sie für Vögel und Säuger bekannt sind (AschоғF 1958), lassen sich nicht finden.

\section{Untersuchungen im Laboratorium}

Die angeführten Befunde haben keinen Hinweis erbracht, daß eine endogene Komponente vorhanden ist. Ein inneres Zeitmeßvermögen miissen die Tiere jedoch besitzen, sonst wären sie nicht zur Sonnenorientierung fähig (PAPI \& PARDI 1959, PAPI 1960, vgl. auch REMMERT 1965).

Unter konstanten Bedingungen (Abb. 6) konnte die endogene Komponente der lokomotorischen Aktivität deutlich erwiesen werden. Wie von einem circadianen 
Rhythmus zu fordern, so erwies sich, daß die Periodenlänge nicht genau 24 Stunden beträgt, sondern bei einer Helligkeit von 1000 Lux etwas größer ist. Die Tiere folgen damit der circadianen Regel Aschoffs $(1957,1958)$, nach der dunkelaktive Arten ihre Periodik mit steigender Intensität des Dauerlichts verlängern. Jedoch zerfiel der erkennbare Rhythmus im Verlauf der folgenden Tage, und gleichzeitig sank die Gesamtaktivität stark ab. Bei Dauerdunkel verschwindet jede Aktivität. Lokomotorische Aktivität konnte in Dauerlicht durch eine kurze Dunkelperiode, in Dauerdunkel durch eine Lichtgabe von 20 min Dauer neu hervorgerufen werden (Abb. 8). Um aktiv zu werden, benötigen die Tiere also den Wechsel von Licht und Dunkelheit.

Die endogene Komponente des Rhythmus kommt bei der lokomotorischen Aktivität nur sehr selten zum Tragen; denn sie wird durch Außenfaktoren völlig ïberlagert.

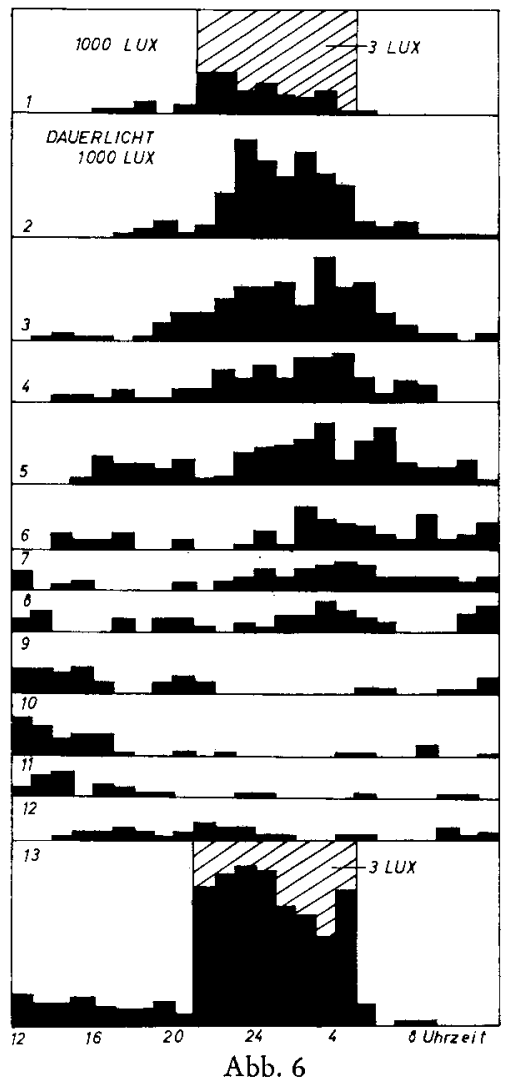

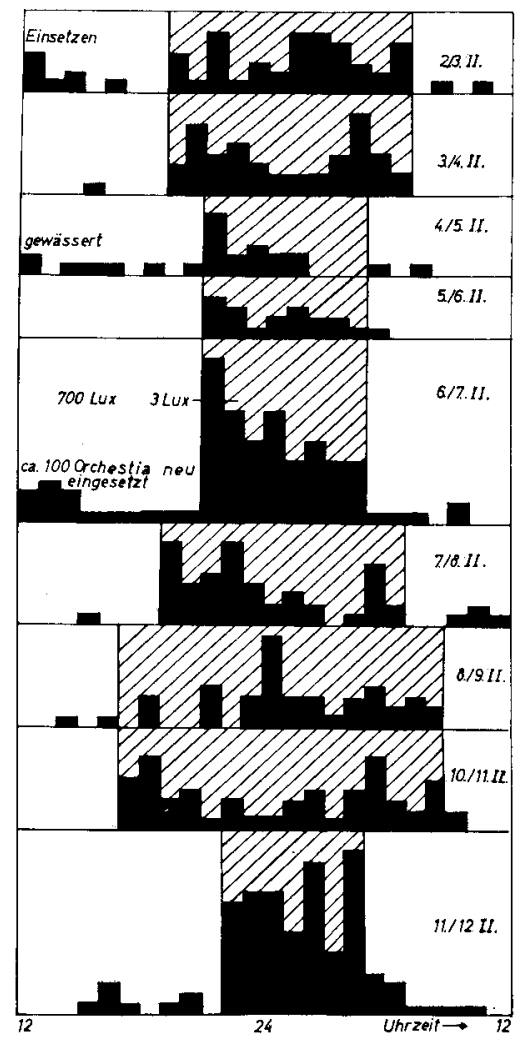

Abb. 7

Abb. 6: Aktivität einer Population (60 Tiere) von Orchestia platensis unter konstanten Bedingungen (Dauerlicht $1000 \mathrm{Lux}, 100 \%$ relative Luftfeuchte, $18^{\circ} \mathrm{C}$ ). Verlängerung der Periodik im Dauerlicht; dann Absinken der Stärke der Aktivität. Ein neuerlicher Licht-Dunkel-Wechsel wird mit starker Aktivität beantwortet

Abb. 7: Aktivität einer Population von Orchestia platensis bei konstanter Temperatur $\left(16^{0} \mathrm{C}\right)$ und $100 \%$ relativer Luffeuchte. Die täglich wechselnden Dunkelzeiten werden ohne Verzögerungen mit Aktivität beantwortet 
Das wurde in einem weiteren Versuch deutlich: Bei konstanter Temperatur $\left(16^{\circ} \mathrm{C}\right)$ und $100 \%$ relativer Lufteuchte wurden den Tieren täglich wechselnde Dunkelzeiten geboten. Die Aktivität folgt diesen wechselnden Bedingungen ohne Verzögerung (Abb.7).

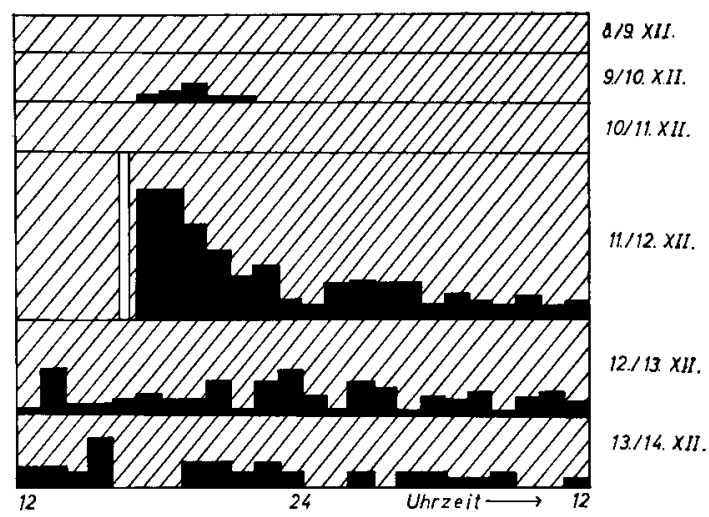

Abb. 8: Absinken der Aktivität von Orchestia platensis (auf null) bei Dauerdunkel. Eine kurze Lichtzeit (20 min) hat starke Aktivität zur Folge

\section{DISKUSSION}

Orchestia platensis lebt bei einer großen Populationsdichte in einem Milieu, welches ihm nur während etwa eines halben Jahres Fortpflanzungsmöglichkeiten bietet, und zwar in der Zeit von April bis Oktober (Bock 1967). Während dieses halben Jahres aber ist das Tier dunkelaktiv. Damit wird seine mögliche Aktivität weiter stark eingeschränkt. Hinzu kommt, daß durch Wind, zu starke Dunkelheit, durch Regen und durch niedrige Temperaturen während vieler Nächte ungünstige Lebensbedingungen herrschen. Im ganzen steht den Individuen dieser Art weniger als etwa $10 \%$ seiner Gesamtlebensdauer für seine aktiven Funktionen zur Verfügung. Eine ungünstige Wirkung können ferner Katastrophen in dem Milieu von Orchestia haben, welche die Amphipoden zu ungünstigen Tageszeiten zur Aktivität bringen und damit hohe Verluste bedingen. Orchestia scheint an diese Verhältnisse dadurch angepaßt zu sein, daß günstige Bedingungen sofort ohne Verzögerung ausgenützt werden können.

Die endogene Komponente des Tagesrhythmus ist zwar im Laboratorium nachweisbar, sie spielt aber im Freiland - im Gegensatz zu den Verhältnissen bei Warmblütern-praktisch keine Rolle. Somit ist ein festgelegtes Muster der Aktivitätsperiodik nicht erkennbar.

\section{ZUSAMMENFASSUNG}

1. Adulte Orchestia platensis (KRÖYER) sind ebenso wie adulte Individuen anderer supralitoraler Talitriden am Sandstrand dunkelaktiv. 
2. In sehr dunklen Nächten, bei Regen, Sturm und niedrigen Temperaturen bleibt Orchestia platensis inaktiv. Fördernd auf die Aktivität wirken helle Nächte (Mond schein), hohe Luffeuchtigkeit, geringe Windgeschwindigkeit und Temperaturen $z$ wischen $10^{\circ}$ und $20^{\circ} \mathrm{C}$.

3. Durch Uberflutung und große Trockenheit kann Aktivität auch am Tage ausgelöst werden (Katastrophenaktivität).

4. Eine endogene Komponente der Aktivität läßt sich unter konstanten Bedingungen im Labor nachweisen. In Dauerlicht wird die Periodenlänge entsprechend der circadianen Regel verlängert.

5. Die Koppelung der Aktivität an die innere Uhr ist jedoch sehr locker; sie wird im Freiland völlig durch Umweltfaktoren überlagert.

6. Damit folgt die Aktivität von Orchestia im Freiland ausschließlich den herrschenden Bedingungen. Der Vorteil eines solchen Verhaltens am instabilen Milieu des Meeresstrandes wird diskutiert.

Die Untersuchungen wurden mit Hilfe einer Sachbeihilfe der Deutschen Forschungsgemeinschaft durchgeführt, die Herrn Prof. Dr. H. Remmert für seine Arbeiten an der biologischen Grenze zwischen Meer und Land zur Verfügung gestellt wurde.

\section{ZITIERTE LITERATUR}

Aschoff, J., 1957. Aktivitätsmuster der T'agesperiodik. Naturwissenschaften 44, 361-367.

- 1958. Tierische Periodik unter dem Einfluß von Zeitgebern. Z. Tierpsychol. 15, 1-30.

Bock, K.-D., 1967. Experimente zur OKologie von Orchestia platensis KröYER. Z. Morph. Okol. Tiere 58, 405-428.

Bünning, E., 1963. Die physiologische Uhr. 2. Aufl, Springer, Berlin, 153 pp.

PApr, F., 1960. Orientation by night: the moon. Cold Spr. Harb. Symp. quant. Biol. 25, $475-480$.

- \& Pard, L., 1959. Nuovi reperti sull'orientamento lunare di Talitrus saltator Montagu (Crustacea-Amphipoda). Z. vergl. Physiol. 41, 583-596.

Remmert, H., 1965. Biologische Periodik. In: Handbuch der Biologie. Begr. von L. von Bertalanffy. Hrsg. von F. Gessner. Akad. Verl. Ges. Athenaion, Frankfurt a. M., 5, 335-411.

ToNGiorgi, P., 1963. Ricerche ecologiche sugli artropodi di una spiaggia sabbiosa del litorale tirrenico. Redia 48, 165-177.

Williams, G., 1958. Mechanical time-sorting of pitfall captures. J. Anim. Ecol. 27, 27-35.

\section{Diskussion im Anschluß an den Vortrag RüPpeLL}

Heydemann: Wir haben in unserer Arbeitsgruppe ebenfalls seit einiger Zeit mit Bodenfallen gearbeitet, die im Litoral eine stundenweise automatische Sonderung der sich aktiv bewegenden epigäischen Tiere gestatten (Hexdemann 1964, Verh. Dt. Zool. Ges., 609). Nebenher wurden in den im Gezeitengebiet der nordwestdeutschen Küste gelegenen Salzwiesen auch Befunde an Orchestia gammarellus und $O$. platensis gewonnen. Sie zeigen, daß in den ausgedehnten, mit Puccinellia maritima und Festuca rubra litoralis bewachsenen Zonen adulte Orchestia gammarellus auch in der Lichtperiode aktiv sind. Sicherlich dürte dabei der Beschattungsgrad unterhalb der Vegetation eine Rolle spielen. Es bleibt dabei natürlich fraglich, ob sich hierin Orchestia gammarellus eventuell anders als $O$. platensis verhält. Deutlich war hier eine $\mathrm{Ab}$ hängigkeit der Aktivität auch von der Höhe der Vegetation (beweidete und unbeweidete 
Areale). Bei Orchestia gammarellus ist also eine deutliche Abhängigkeit der von Ihnen geschilderten Aktivitätsperiodik von verschiedenen Biotoptypen und Biotopvarianten zu beobachten.

RüPPELL: Im Freiland wird die Tagesrhythmik vorwiegend durch die exogene Komponente geregelt. Ihre Beobachtungen passen daher recht gut zu meinen Befunden.

HeYdemann: Sie sprachen von einer "Katastrophenaktivität" der Orchestia platensis in der Anwurfzone bei Aufkommen der Flut. Eine solche Aktivität ist nach unseren bisherigen Befunden in den Salzwiesen der Nordseeküste bei Orchestia gammarellus, aber auch bei Orchestia platensis, weniger ausgebildet. In den ausgedehnten Salzwiesenbeständen dürfte der Fluchtaktivität auch nicht die Bedeutung zukommen, welche sie am schmalen Sandstrand mit seinem stärkeren Gefälle besitzt. Die Verbreitung von Orcbestia platensis und O. gammarellus in den Salzwiesen der Nordseeküste muß in diesem Zusammenhang noch aufgeklärt werden. In manchen Arealen kamen im gleichen Biotop nur O. gammarellus vor, in anderen nur O. platensis.

PAFFENHÖFER; Existiert neben einer Licht-Dunkel-Aktivität audh eine unterschiedliche Aktivität zu verschiedenen Jahreszeiten?

RüppeLL: Nein; es verschiebt sich allerdings $z$ wangsläufig der zur Lokomotion bevorzugte Bereich der Temperatur. Orchestia ist in Winter noch bei Temperaturen aktiv, die im Sommer aktivitätslähmend wirken.

Amaniev: Le Monsieur Rüppell a-t-il constaté des différences d'activité en fonction (1) des couches d'âge d'une population homogène. Les jeunes, ont-ils ou non la même activité que les adultes? (2) d'un rythme interne lié aux cycles reproducteurs?

RüppelL: Mein Referat bezog sich nur auf adulte Individuen; junge verhalten sich anders. Im Jahreszyklus kommen höchstens quantitative Unterschiede der Akrivität vor. Diese sind mit meiner Methode nicht erfaßbar.

WIESER: Sind Ihre experimentellen Hell-Dunkel-Veränderungen formal Rechteckschwingungen oder sinusförmige Schwingungen gewesen? Es wäre vielleicht nicht unwichtig, die Auslösewirkung dieser beiden Reizformen zu vergleichen.

RüPPELL: Im Experiment wurden nur Rechteckschwingungen verwendet. 